\title{
Use of Polycrystalline Ice for Assembly of Large Area Au Nanoparticle Superstructures as SERS Substrates
}

\author{
Deribachew Bekana, ${ }^{\dagger \neq}$ Rui Liu, ${ }^{\dagger}$ Meseret Amde, ${ }^{\dagger,}$ and Jing-Fu Liu ${ }^{*}, \dagger$, \\ ${ }^{\dagger}$ State Key Laboratory of Environmental Chemistry and Ecotoxicology, Research Center for Eco-Environmental Sciences, Chinese \\ Academy of Sciences, P.O. Box 2871, Beijing 100085, China \\ ${ }^{\ddagger}$ University of Chinese Academy of Sciences, Beijing 100049, China
}

Supporting Information

ABSTRACT: It is still a great challenge to develop simple and low-cost methods for preparation of surface-enhanced Raman scattering (SERS) substrates with high sensitivity and reproducibility. Taking advantage of the microstructure of polycrystalline ice, we developed a new method to assemble large area gold nanoparticle (AuNP) superstructures as SERS substrates without external templating and aggregating agent. The assembly was conducted by freezing AuNP colloid at -20 ${ }^{\circ} \mathrm{C}$, which concentrated AuNPs in the ice veins and produced an AuNP superstructure upon thawing the ice. The AuNP superstructures exhibited high SERS activity with enhancement factors on the order of $7.63 \times 10^{7}$ owing to the high-

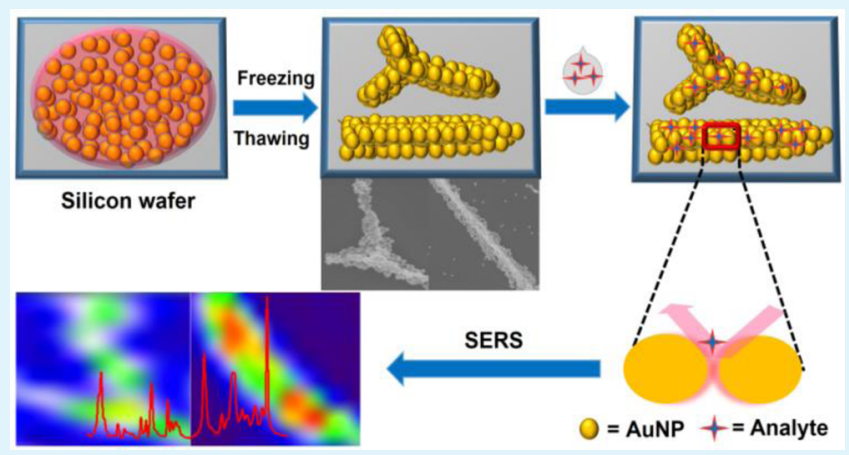
density hot spots throughout the superstructures. The SERS activity was found to increase with particle size and aggregate size of AuNP superstructures. Besides, the substrates showed good uniformity and reproducibility with relative standard deviations of $11.9 \%$ and $12.4 \%$, respectively. The substrates showed longterm stability, maintaining SERS activity over a period of five months without noticeable change in morphology of the superstructures. The substrates was further used for label-free detection of trace Thiram on apple fruit with high sensitivity down to the concentration of $0.28 \mathrm{ng} / \mathrm{cm}^{2}$, offering great potential to monitor Thiram levels in foodstuffs and environmental samples.

KEYWORDS: self-assembly, superstructure, polycrystalline ice, microstructure, veins, aggregate size

\section{INTRODUCTION}

Over the past few decades, significant advancement has been achieved in the application of surface-enhanced Raman scattering (SERS) as an analytical tool. This is owed to its fingerprint spectra provision capacity for single-molecule detection, as well as other decisive advantages such as ease of use, instrument miniaturization, and minimized sample preparation over other analytical methods. ${ }^{1-4}$ So far, SERS has been successfully used in various areas like in situ chemical reaction monitoring ${ }^{5,6}$ and detection of environmental contaminants. $^{1,7,8}$

The unprecedented sensitivity of SERS arises from the million-fold amplification of the Raman signal of target analytes that are adsorbed or positioned in close proximity of noble metal (mostly $\mathrm{Au}$ and $\mathrm{Ag}$ ) nanostructures. ${ }^{3}$ This enhanced Raman signal arises from intense electromagnetic (EM) field generated by excitation of collective oscillations of the free surface electrons, the so-called physical-enhancement mechanism, as well as via formation of charge-transfer complexes between linked analytes and SERS substrates, known as the chemical-enhancement mechanism. ${ }^{9}$ The SERS activity is highly dependent on the light absorption/scattering, which changes with the morphology and geometric arrangement of the noble metal nanostructures employed as SERS substrates. ${ }^{10}$
The key for practical application of SERS as an analytical tool is to fabricate SERS substrates that can simultaneously produce high-density and uniform hot spots to offer strong SERS enhancement and reproducible SERS signals. In this regard, highly ordered metal nanostructures such as three-dimensional superstructures and superlattices of nanoparticles (NPs) have been reported as effective SERS substrates. ${ }^{2,3}$ It is of paramount importance to assemble metal NPs into ordered superstructures as NP assemblies possess spatially delocalized regions that support highly intense local EM fields called hot spots, which are distinctly different from their respective isolated NPs. ${ }^{11}$ This arises from the plasmonic/electromagnetic coupling effect induced between closely spaced adjacent NPs at nanogap junctions. $^{12-14}$ In such an assembly, with decreasing interparticle distance $<10 \mathrm{~nm}$, high SERS enhancement factor on the order of $\geq 10^{7}$ was achieved. ${ }^{12,14-16}$ In general, the advantages of NP superstructure as SERS substrates are due to their unique morphology, which offers plentiful and uniformly distributed hot spots, and their large surface area for adsorption of more target molecules, contributing to strong SERS

Received: November 30, 2016

Accepted: December 16, 2016

Published: December 16, 2016 
enhancement. ${ }^{2}$ However, many of the SERS substrates reported in the literature lack some of the aforementioned characteristics. For example, some SERS substrates showed uneven distribution of hot spots, and only $\sim 0.01 \%$ of the total sites that are known as SERS hot spots accounts for the recorded Raman signals. ${ }^{17}$ Therefore, development of SERS-based analytical methods calls for preparation of efficient SERS substrates with plentiful and uniformly distributed hot spots to ensure high sensitivity and SERS signal reproducibility. ${ }^{2}$

Currently, there are two general routes for the preparation of AuNP assemblies/aggregates as SERS substrates: either through induced aggregation of prior synthesized AuNPs by addition of an external agent or via in situ synthesis of NP aggregates. ${ }^{13}$ Assembling metal NPs can be achieved using macromolecules and/or polymers as a template to assemble NPs into ordered structures ${ }^{14,18-20}$ or using different crosslinkers, capping agents, and an aggregation-inducing agent to get noble metal assemblies. ${ }^{15,21-23}$ However, the presence of external agent/polymeric linkers might have adverse effects on SERS performance such as interfering with SERS detection of trace analyte. ${ }^{24}$ Thus, it is highly desired to assemble SERS substrates without external agent/polymeric linkers.

Polycrystalline ice (PCI) was reported to direct assembly of colloidal nanostructures into one-dimensional (1D) chains. ${ }^{25}$ In this assembly the aqueous solution of colloidal AuNPs was incubated below the freezing point at $-20^{\circ} \mathrm{C}$, and the AuNPs were rejected as impurities due to their limited solubility in ice crystals and therefore concentrated and packed together between growing ice crystals. ${ }^{25,26}$ It is expected that this approach can be extended to prepare nanogap-rich, large area, densely arranged, and closely packed AuNP superstructures, which could generate SERS hot spots as effective SERS substrates without external/polymer cross-linkers.

The aim of the current study was to take advantage of the microstructure of PCI to develop a simple, rapid, and low-cost strategy for assembling SERS active substrates without external templating and aggregating agent. The added value of this method is that it produces architecturally defined AuNP superstructures that generate high-density and uniform hot spots over the surface of the substrate, demonstrating strong SERS enhancement and signal reproducibility. Moreover, the strategy employed no external templating and aggregating agent, making SERS detection background interference-free, and the assembling is low-cost and would not require highly trained personnel. Therefore, the current study is interesting as the low-cost SERS substrate preparation method is the most important requirement for practical application while maintaining sufficient high signal enhancement. In addition, to the best of our knowledge, there has been no report on the use of polycrystalline ice for the preparation of SERS substrates, although the method has been reported for assembly of $1 \mathrm{D}$ structures.

The parameters influencing the SERS activity of prepared AuNP superstructures, such as the size of the AuNPs and the aggregate size of the formed AuNP superstructures, were optimized using crystal violet $(\mathrm{CV})$ as Raman probe. The reallife practicability of the developed SERS substrates was demonstrated by detecting trace Thiram eluted from the apple fruit surface (Scheme 1).

\section{EXPERIMENTAL SECTION}

2.1. Chemicals and Materials. Tetrachloroauric acid trihydrate $\left(\mathrm{HAuCl}_{4} \cdot 3 \mathrm{H}_{2} \mathrm{O}, 99 \%\right), \mathrm{CV}(90 \%)$, nitric acid $\left(\mathrm{HNO}_{3}, 65 \%\right)$, and
Scheme 1. Illustration of Assembly of AuNP Superstructures Assisted by Microstructure of Ice; AuNP Color Change in the Photos on the Right Represents Formation of AuNP Assemblies

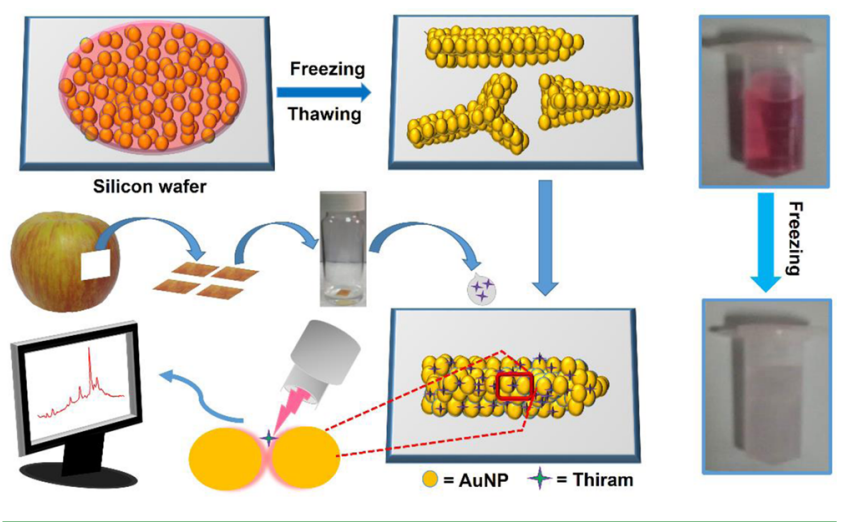

hydrochloric acid ( $\mathrm{HCl}, 38 \%)$ were purchased from Shanghai Sinopharm Chemical Reagent Co. Ltd. (Shanghai, China). Thiram (99.9\%) and trisodium citrate $\left(\mathrm{Na}_{3} \mathrm{C}_{6} \mathrm{H}_{5} \mathrm{O}_{7}, \geq 99 \%\right)$ were purchased from Sigma-Aldrich (Darmstadt, Germany). Silicon wafer was purchased from Xin Xin Bai Ri Technology Co. (Beijing, China). All chemicals were of the analytical grade. Ultrapure water (18.2 M $\Omega$ ) purified by Milli-Q gradient quality system (Millipore, Bedford, MA, U.S.A.) was used throughout the experiment. All glassware used was cleaned by soaking in aqua regia solution $\left(\mathrm{HNO}_{3} / \mathrm{HCl}, 1: 3\right)$, followed by thorough rinsing with ultrapure water prior to use.

2.2. Synthesis of AuNPs. AuNPs with particle size of $18 \mathrm{~nm}$ were synthesized by citrate-reduction method according to the literature. ${ }^{27}$ Briefly, an aqueous solution of $\mathrm{HAuCl}_{4} \cdot 3 \mathrm{H}_{2} \mathrm{O}(100 \mathrm{~mL}, 0.25 \mathrm{mM})$ was refluxed in oil bath for $10 \mathrm{~min}$ with vigorous stirring, followed by adding $\mathrm{Na}_{3} \mathrm{C}_{6} \mathrm{H}_{5} \mathrm{O}_{7}(3 \mathrm{~mL}, 1 \%, \mathrm{w} / \mathrm{w})$ into the solution. The mixture was heated for $30 \mathrm{~min}$ before cooling down to room temperature, then purified by centrifugation $(11000 \mathrm{~g}, 30 \mathrm{~min})$ twice to remove the excess sodium citrate, and redispersed in ultrapure water. The AuNPs with larger particle size were synthesized using the modified seedmediated approach method using $18 \mathrm{~nm}$ AuNPs as seeds and controlling the concentration of sodium citrate in the reaction mixture. Briefly, the aqueous solution of $\mathrm{HAuCl}_{4} \cdot 3 \mathrm{H}_{2} \mathrm{O}(100 \mathrm{~mL}, 0.25 \mathrm{mM})$ was refluxed for $10 \mathrm{~min}$ with vigorous stirring, followed by adding seeds $(18 \mathrm{~nm}, 8 \mathrm{~mL})$ and different volumes of trisodium citrate $\left(\mathrm{Na}_{3} \mathrm{C}_{6} \mathrm{H}_{5} \mathrm{O}_{7}, 1 \% \mathrm{w} / \mathrm{w}, 750-250 \mu \mathrm{L}\right)$. Then, the mixture was heated for $30 \mathrm{~min}$ before cooling down to room temperature followed by purification by centrifugation.

2.3. Assembly of AuNP Superstructures. The assembly was prepared by freezing the aqueous solution of the synthesized colloidal AuNPs according to the reported method. ${ }^{25}$ First, silicon wafer $(1 \mathrm{~cm}$ $\times 1 \mathrm{~cm}$ ) was cleaned with ethanol repeatedly followed by water, and then it was air-dried. Then $20 \mu \mathrm{L}$ of AuNP colloidal solution was dropped on the chip of silicon wafer and placed in the refrigerator at $-20{ }^{\circ} \mathrm{C}$ for $20 \mathrm{~min}$ to ensure ice formation. Then the sample was kept at room temperature until it was completely dried and ready for use as a SERS substrate. The reference sample was prepared by dropping 20 $\mu \mathrm{L}$ of AuNP colloidal solution on the chip of silicon wafer and airdrying at room temperature.

2.4. Characterization. Transmission electron microscopy (TEM; H-7500, Hitachi) and field emission scanning electron microscopy (FESEM; Su-8020, Hitachi) were used to characterize the morphologies of synthesized AuNPs and assembled AuNP superstructures. Samples for TEM were prepared by dropping $\sim 5 \mu \mathrm{L}$ of the colloidal solution onto a carbon-coated copper grid, which was dried and then examined by TEM. The FESEM images of AuNP superstructures were acquired directly as assembled on the silicon wafer.

2.5. SERS Measurement. The samples for SERS measurement of $\mathrm{CV}$ were prepared by loading $10 \mu \mathrm{L}$ of different concentrations $(1 \times$ 


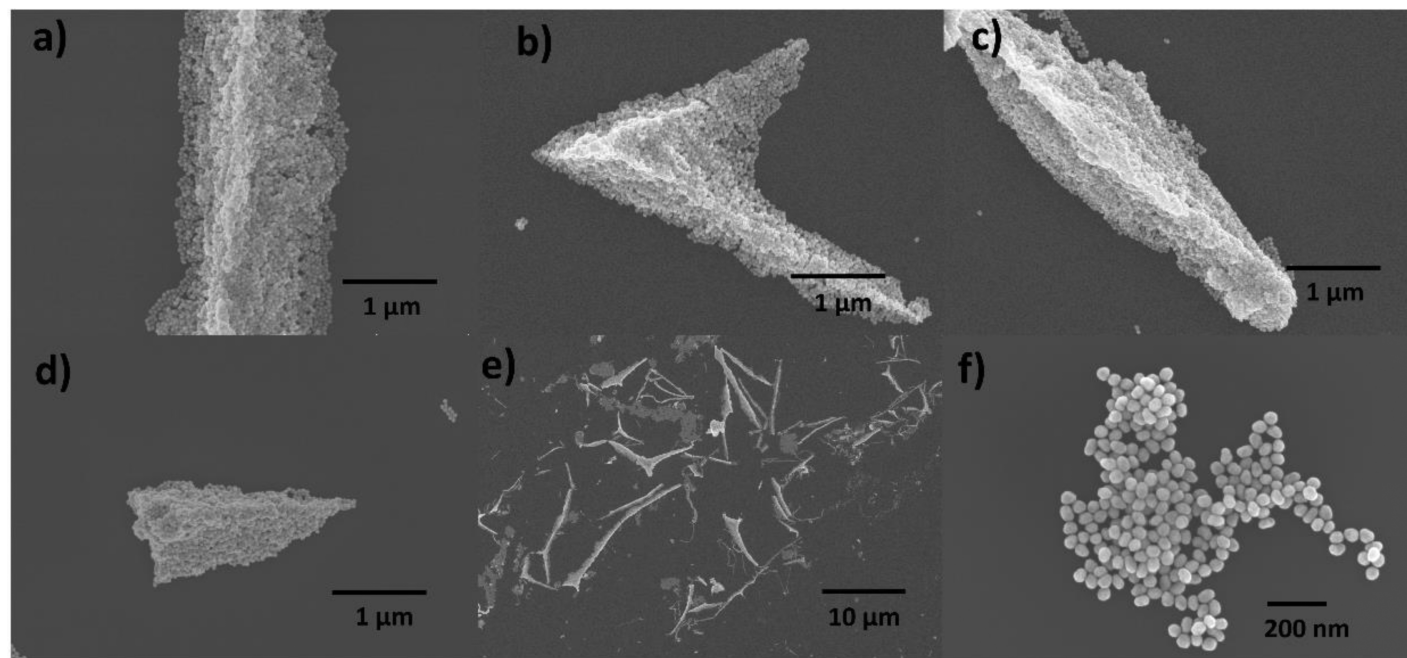

Figure 1. FESEM images of the assembled AuNP superstructures as SERS substrates. (a-d) Large area AuNP superstructures of different microstructure. (e) Low-magnification SEM images of the AuNP superstructures produced through the current method showing formation of large area superstructures as major product. (f) SEM image of unassembled/unfrozen AuNPs as a reference.
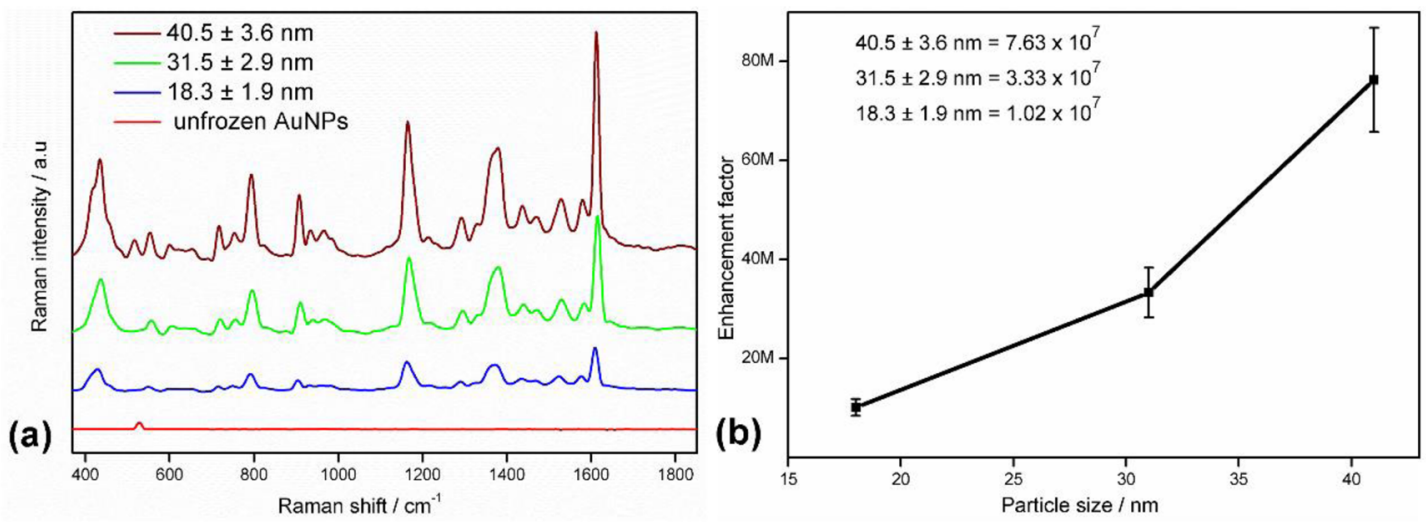

Figure 2. SERS activity of superstructures assembled from different sized AuNPs. (a) SERS spectra of CV $\left(1 \times 10^{-7} \mathrm{M}\right)$ loaded on AuNP superstructures with different particle sizes and unfrozen AuNPs. (b) Plot of SERS enhancement factor at $1621 \mathrm{~cm}^{-1}$ as a function of particle size of AuNPs in the assemblies.

$10^{-7}$ to $\left.1 \times 10^{-10} \mathrm{M}\right)$ of $\mathrm{CV}$ ethanolic solution on the AuNP superstructures and then leaving them at room temperature for $\sim 5$ min to evaporate the solvent. After that, the SERS spectra were collected using a Renishaw Invia Raman microscope (New Mills, U.K.) equipped with excitation wavelength of $633 \mathrm{~nm}, 50 \times$ objective, and laser power of $0.1 \mathrm{~mW}$ with $10 \mathrm{~s}$ exposure time. Quantitative measurements for $\mathrm{CV}$ were carried out with $1 \mathrm{~s}$ integration time. The SERS measurement of Thiram was conducted in the same way except that a series of concentrations $\left(1 \times 10^{-4}\right.$ to $\left.1 \times 10^{-7} \mathrm{M}\right)$ of Thiram in ethanol were prepared and $0.5 \mathrm{~mW}$ laser power was adopted.

To quantitatively evaluate the SERS enhancement for each of the assemblies, the SERS enhancement factor (EF) was calculated by eq 1,2

$$
\mathrm{EF}=\left(I_{\mathrm{SER}} / N_{\mathrm{SERS}}\right) /\left(I_{\mathrm{NR}} / N_{\mathrm{NR}}\right)
$$

where $I_{\text {SERS }}$ denotes the SERS intensity of CV adsorbed on the AuNP superstructures; $I_{\mathrm{NR}}$ is the normal Raman intensity of aqueous solution of CV dried on the Si wafer; and $N_{\text {SERS }}$ and $N_{\mathrm{NR}}$ stand for the number of probe molecules contributing to the SERS signal and the normal Raman signal, respectively.

2.6. Sample Analysis. Apple fruit was purchased from a local market. For the detection of Thiram fungicide on apple fruit, prior to washing the apple fruit was peeled and cut into $1 \mathrm{~cm}^{2}$. Initially, the blank sample was prepared by placing apple peels in $500 \mu \mathrm{L}$ of ethanol to extract trace Thiram from the surface. To validate the method, the apple peels were spiked by loading $5 \mu \mathrm{L}$ of different concentrations of Thiram solution in ethanol onto the samples and were completely dried at room temperature for $10 \mathrm{~min}$. Then, the spiked apple peels were placed in the required volume of ethanol and extracted to final Thiram concentrations ranging from $5 \times 10^{-5}$ to $1 \times 10^{-7} \mathrm{M}$. Thiram series in ethanol solution concentrations in the range of $1 \times 10^{-5}$ to 1 $\times 10^{-7} \mathrm{M}$ were used as standard solutions. Then, $10 \mu \mathrm{L}$ of each concentration of eluted Thiram and standard solution were dropped onto the AuNP superstructures and were completely dried for $5 \mathrm{~min}$ followed by SERS spectra acquisition (Scheme 1).

\section{RESULTS AND DISCUSSION}

3.1. Characterization of the As-Synthesized AuNPs. The concentration and particle size of as-synthesized AuNPs were characterized using TEM techniques. The average particle sizes of the three as-synthesized AuNPs estimated from TEM images using Nano Measurer software (v. 1.2) by counting more than 200 particles (Figure S1) were $18.3 \pm 1.9,31.5 \pm$ 2.9 , and $40.5 \pm 3.6 \mathrm{~nm}$; their concentrations calculated using the TEM result based on the reported method ${ }^{28}$ were $5.05 \times$ $10^{13}, 9.91 \times 10^{12}$, and $4.66 \times 10^{12}$ particles $/ \mathrm{mL}$, respectively. The details are shown in the Supporting Information.

3.2. Assembly and Characterization of AuNP Superstructures. The AuNP superstructures was assembled by 

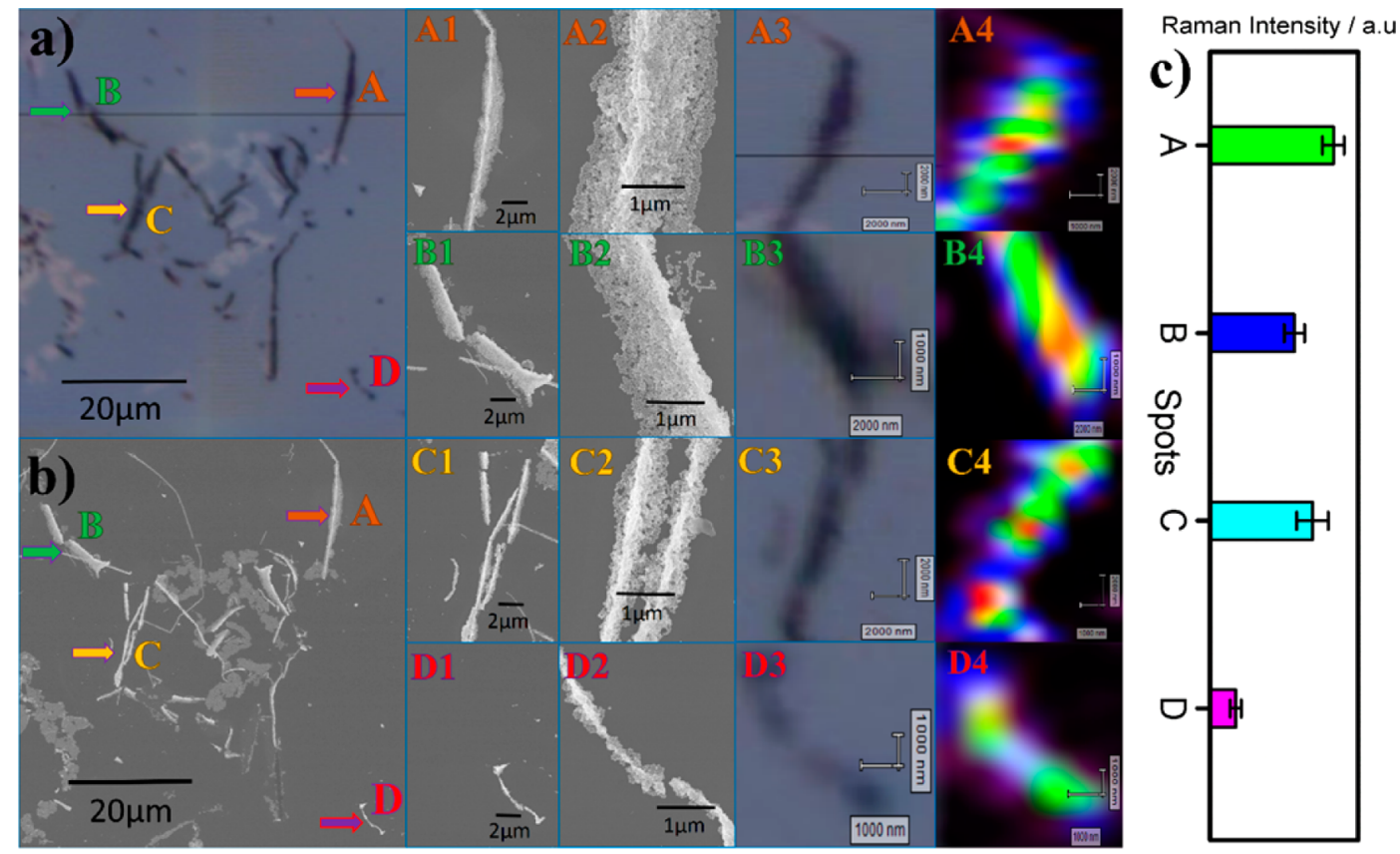

Figure 3. Correlation of SERS activity with aggregate size of AuNP superstructures. Optical (a) and SEM (b) images of selected area for Raman mapping. A1 and A2, B1 and B2, C1 and C2, and D1 and D2 show the SEM images and magnified SEM images of the constituent of AuNP superstructures, respectively; A3 and A4, B3 and B4, C3 and C4, and D3 and D4 show the optical and Raman mapping images at $1621 \mathrm{~cm}^{-1}$ for spots A, B, C, and D, respectively. (c) Variation of SERS signals intensity at $1621 \mathrm{~cm}^{-1}$ for comparison of SERS performance.

freezing the as-synthesized colloidal AuNP solution at $-20{ }^{\circ} \mathrm{C}$, and the assemblies are shown in Figure 1. As can be evidenced from the FESEM images (Figure $1 \mathrm{a}-\mathrm{d}$ ), freezing colloidal AuNPs and thawing produced large area AuNP superstructures accompanied by very few simple 1D structures (Figure S2). During the freezing process, a system of interconnected waterfilled veins are formed in PCI, which lie along lines where ice grain meets. ${ }^{29}$ In PCI, in the process of ice formation the NPs are expelled out and concentrate near the boundaries of growing ice grains, and thawing the formed ice leaves densely arranged AuNP superstructures confined to the triangular prism shape of the veins. Unlike in a previous report, ${ }^{25}$ the major products of the current assemblies are large area AuNP superstructures that are composed of densely arranged and closely packed AuNPs (Figure 1e). This is ascribed to the high concentration of AuNP colloids used for the assembly and, hence, the presence of a sufficient quantity of AuNPs, allowing the constituent AuNPs to fill the entire vein at the final stage of freezing. Thus, formation of larger ice grains at the final stage of freezing concentrates more NPs at the grain boundaries, giving rise to formation of large area AuNP superstructures exhibiting the shape of the veins. ${ }^{25}$ Such assemblies should be excellent SERS substrates owing to their nanogap-rich structures that could generate high-density hot spots.

\subsection{SERS Activity of Superstructures Assembled from} Different Sized AuNPs. SERS activity of the prepared nanogap-rich AuNP superstructures was evaluated by using $\mathrm{CV}$ as Raman probe. ${ }^{9}$ Figure 2 shows the SERS spectra of CV $\left(1 \times 10^{-7} \mathrm{M}\right)$ loaded on the AuNP superstructures assembled from different particle sizes $(18.3 \pm 1.9,31.5 \pm 2.9$, and $40.5 \pm$ $3.6 \mathrm{~nm}$ ), in which apparent Raman bands at 802, 918, 1178, 1376,1588 , and $1621 \mathrm{~cm}^{-1}$ ascribed to the main vibrational bands of CV were observed. The Raman band assignments are made based on the data reported in the literature. ${ }^{9}$ Remarkable SERS signal enhancement was observed for CV adsorbed on the AuNP superstructures as compared to unassembled/ unfrozen AuNPs (Figure 2a), demonstrating the formation of plentiful hot spots.

The influence of particle size on SERS enhancement was quantitatively evaluated by plotting intensity of Raman signal at $1621 \mathrm{~cm}^{-1}$ as a function of particle size of AuNPs in the assemblies (Figure 2b). The SERS EF increased with particle size, which is similar to previous reports and was ascribed to the huge amplification of the EM field for larger particles, therefore leading to higher SERS enhancement. ${ }^{30}$ It is known that SERS activity increases with increasing particle size and is governed by the EM field effect, which also depends on photon scattering and radiation damping. The increase in local EM field until a certain particle size (optimal size) of metal nanoparticles led to strong SERS enhancement. However, when the particle size is too big (above a certain optimal size), this behavior no longer persists due to radiation damping. ${ }^{31}$ Thus, in current assemblies, as the particle size of coupling adjacent AuNPs increases, large local fields can be induced, producing a highly intense electromagnetic field for larger particle size. However, the largest particle size $(40.5 \pm 3.6 \mathrm{~nm})$ used in the current study is not the optimal particle size, and further studies are needed to establish the optimal particle size in NP assemblies for maximum SERS enhancement.

Similar trends have been observed in previous studies in which $\mathrm{AuNP}^{32}$ and $\mathrm{Au} / \mathrm{AgNP}^{30}$ assemblies with larger particle sizes, 56 and $90 \mathrm{~nm}$, respectively, produced stronger SERS enhancement. Other studies on single particles also observed a direct correlation between SERS activity and particle size, ${ }^{31,33}$ which was attributed to the enhanced EM field around the larger AgNP. ${ }^{31}$ On the contrary, another group reported a decrease in SERS enhancement as the particle size increase. ${ }^{34}$ Such a correlation could be explained due to the formation of large aggregates or agglomerates leading to an overlying layer, thus reducing the coupling between adjacent NPs, which in 

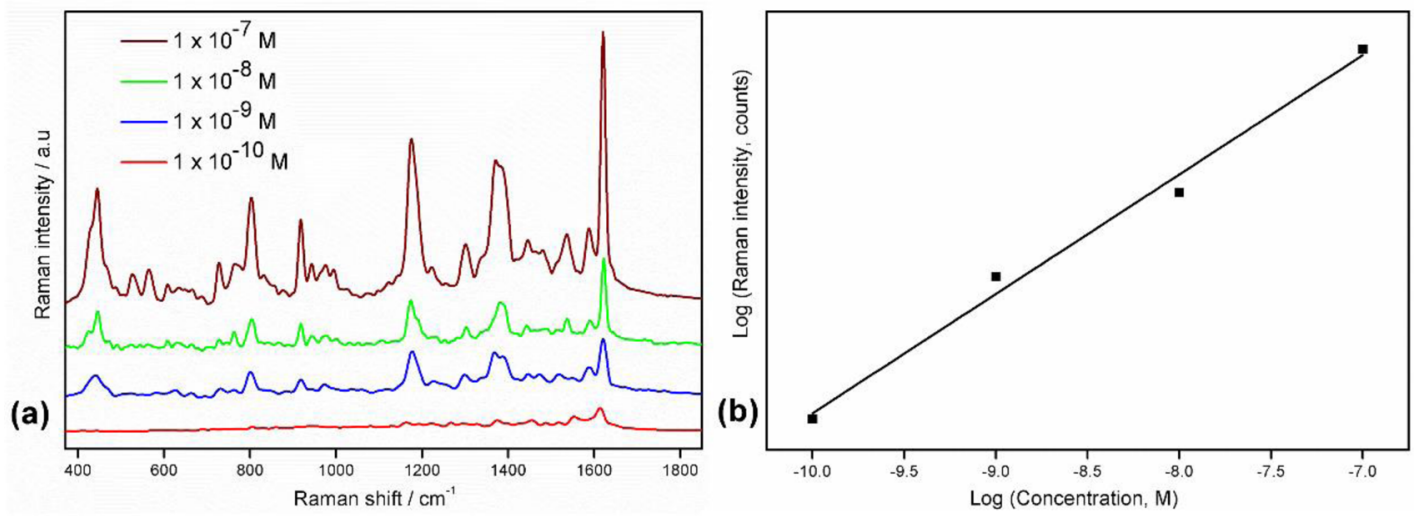

Figure 4. SERS response of $\mathrm{CV}$ at different concentrations. (a) SERS spectra of series concentrations of CV dropped on SERS active AuNP superstructures. (b) Log-log plot of intensity of SERS signals at $1621 \mathrm{~cm}^{-1}$ as a function of concentration of CV.
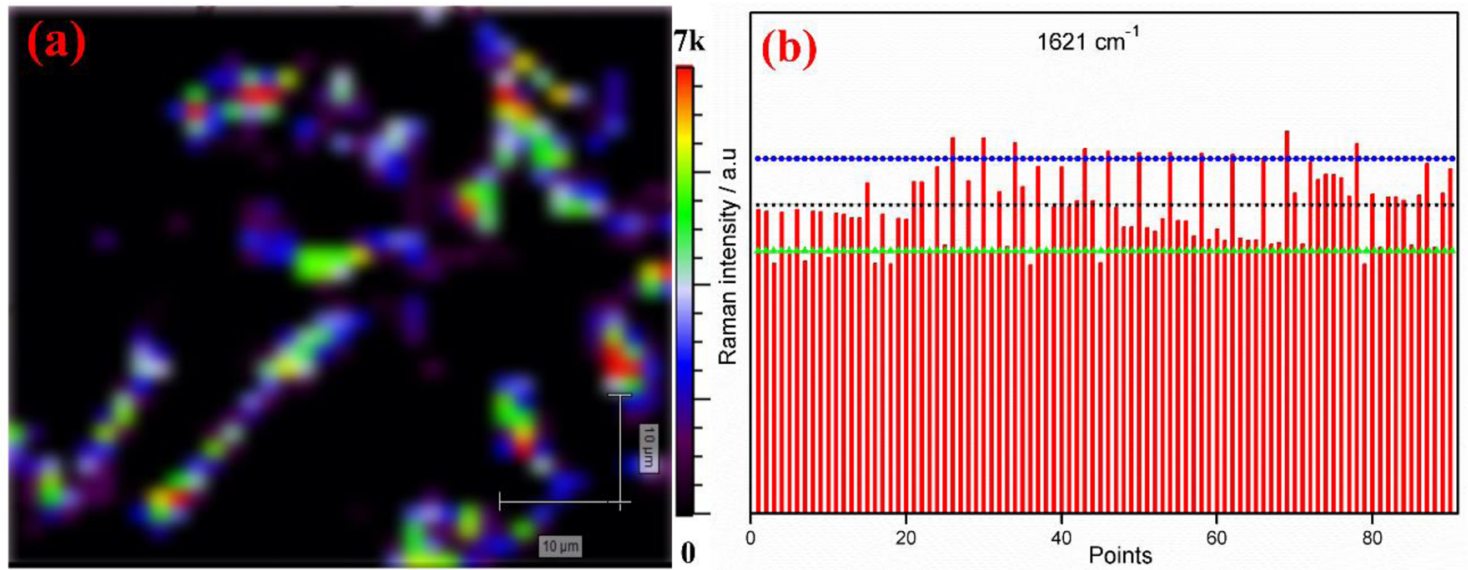

Figure 5. Uniformity of the as-prepared SERS substrates. (a) Raman mapping image at the SERS signal of $1621 \mathrm{~cm}^{-1}$. (b) Variation of intensity of SERS signals at $1621 \mathrm{~cm}^{-1}$. Middle dashed line indicates average, while the top and bottom lines indicate $\pm 15 \%$ of average of intensity of SERS signals $\left(1621 \mathrm{~cm}^{-1}\right)$.

turn decreases the EM field enhancement. ${ }^{35}$ In subsequent studies, an AuNP with a particle size of $40.5 \pm 3.6 \mathrm{~nm}$ was used.

3.4. SERS Activity of Different Superstructures Assembled from the Same AuNPs. The SERS activity of the assembled superstructures of the same sized AuNPs was further evaluated regarding the difference in size, dimensions, and geometries of NP aggregates that were reported to influence the SERS performance. ${ }^{2,15,36}$ To this end, the location with representative microstructures, AuNP superstructures with varying aggregate size, was selected, as shown in the optical image (Figure 3a) and SEM images (Figure 3b). Then, their corresponding SERS activities were compared based on the Raman mapping recorded via spot by spot and the relative SERS signal intensity at $1621 \mathrm{~cm}^{-1}$ (Figure 3c). As can be seen from the plot (Figure 3c), the intensity of the SERS signal varies with microstructures of the AuNP superstructure. Spot A with relatively larger aggregate size showed the greatest SERS signal intensity followed by spots $\mathrm{C}$ and $\mathrm{B}$, while spot $\mathrm{D}$ with smaller aggregate size showed the lowest SERS intensity. It is noteworthy that the microstructure shown in spot D rarely occurred, and we present it here only to evaluate the effect of the aggregate size on the SERS effect under current measurement conditions. The strong SERS enhancement for larger aggregate size was ascribed to the possible formation of more nanogaps by a large number of NPs driven together and creating more hot spots with respect to their size and geometry. ${ }^{15,36,37}$ As can be seen from Figure 3, the geometries of the superstructures in spots $\mathrm{A}$ and $\mathrm{C}$ are slightly different from that in spot B. The superstructures in spots $\mathrm{A}$ and $\mathrm{C}$ exhibited a triangular prism shape, while that in spot B is slightly more flat-faced. In addition, it is believed that the physical orientation of the superstructures relative to the irradiated laser light can affect the detection of optimum signal strength, which might also affect the SERS enhancement. This might dampen the SERS signal intensity of the superstructure shown in spot B due to its aggregate geometry. Also, it has been reported that multidimensional structures are advantageous for having extra hot spots along their dimensions as well as the large area superstructures, allowing adsorption of more target molecules. ${ }^{2}$ The obtained results are in good agreement with previous reports which demonstrated that aggregates with more layers of stacked NPs exhibit greater SERS enhancement. ${ }^{15,23,36}$ It is also noteworthy that major products of current assemblies had comparable microstructures and showed minimal variation in SERS signal intensity, making the superstructures prepared by this procedure potential substrates for SERS detection. It is believed that the overall SERS signal intensity is the sum of signals from all analyte molecules under the irradiated laser beam of detection area. ${ }^{3}$ Therefore, as major products of assemblies produced through the current method, which are used as SERS substrates, had widths greater than the laser spot size $(\sim 1 \mu \mathrm{m})$ of confocal Raman, the number of molecules in 

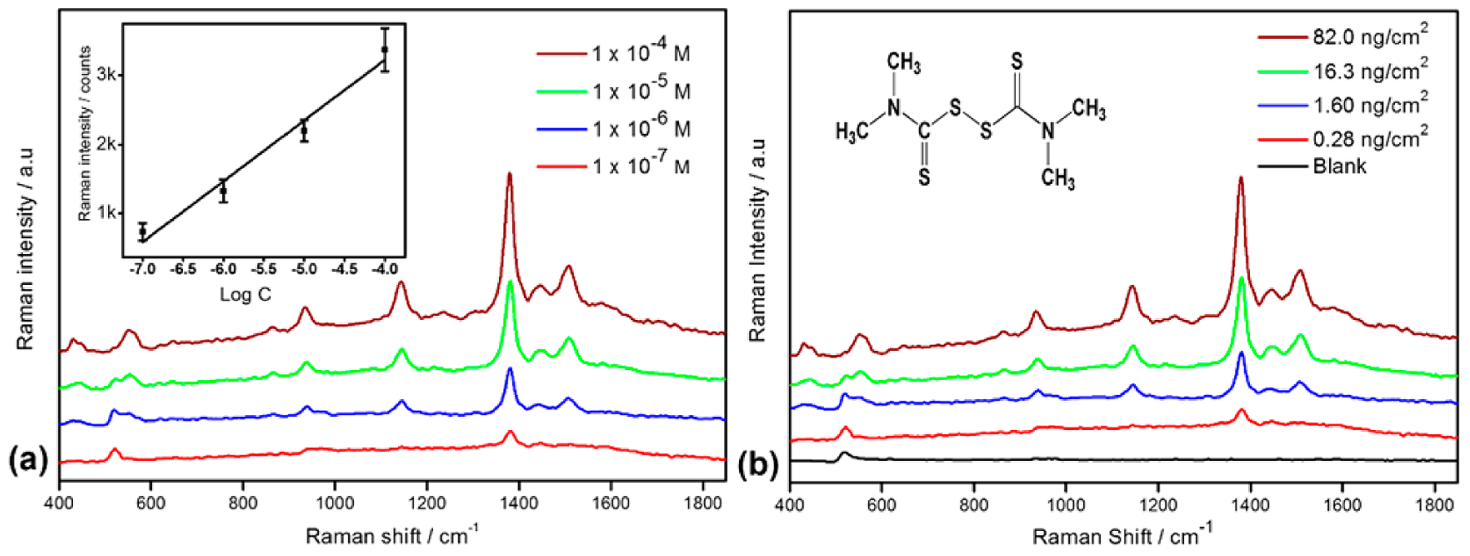

Figure 6. (a) SERS spectra of a series of Thiram standard solutions at various concentrations (M). Inset: Plot of intensities of SERS signals at 1380 $\mathrm{cm}^{-1}$ as a function of the logarithm of concentrations (M) of Thiram. (b) SERS spectra of Thiram eluted from spiked apple peels. Inset: Chemical formula of Thiram fungicide.

the laser irradiated area is believed to be constant and, thus, does not affect the Raman intensity comparison. However, the minor product presented here to evaluate its SERS activity showed low signal enhancement under current measurement conditions. Thus, it was not considered as a SERS substrate that can be easily distinguished from larger superstructure under Leica optical microscope during Raman measurement so that the claimed results throughout the manuscript are repeatable.

3.5. Analytical Performance of the Developed Substrate. Analytical performance of the assembled AuNP superstructures, such as sensitivity, reproducibility and longterm stability for $\mathrm{CV}$, was investigated. SERS spectra of series concentrations of $\mathrm{CV}\left(1 \times 10^{-7}\right.$ to $\left.1 \times 10^{-10} \mathrm{M}\right)$ loaded on the as-prepared substrate (Figure $4 \mathrm{a}$ ) showed that the intensities of SERS signal increased with the CV concentration. The limit of detection (LOD), the lowest concentration at which the most intense signal $\left(1621 \mathrm{~cm}^{-1}\right)$ remained noticeable, was as low as 1 $\times 10^{-10} \mathrm{M}$, endorsing the high sensitivity of the SERS platform. The correlation between concentration of CV and intensity of SERS signal, examined via a log-log plot of intensity of SERS signal versus concentration of $\mathrm{CV}$, exhibited a good linear relationship (Figure $4 \mathrm{~b}$ ). Therefore, such sensitivity of the SERS platform is a promising candidate for detection of other organic pollutants.

The uniformity of the as-prepared substrates was demonstrated via recording spot-to-spot Raman mapping (Figure 5a) on a randomly selected large area containing $>10$ AuNP superstructures produced on the selected area of the substrates. As shown in Figure 5, uniform SERS hot spots were created along the line of the produced AuNP superstructures. The SERS spectra collected from 90 spots showed consistent SERS signal intensification with RSD values of $11.9 \%$ (Figure $5 \mathrm{~b}$ ). The obtained RSD value shows uniformity of hot spots over large area among superstructures produced, and thus, consistent uniformity can be found on the entire surface of as-prepared SERS substrates. In addition, the uniformity of hot spots created across the surface of a single AuNP superstructure was evaluated using 30 SERS spectra collected from random spots via recording spot-to-spot Raman mapping (Figure S3b), which showed precise $(\mathrm{RSD}=11.0 \%)$ SERS signal intensification at $1621 \mathrm{~cm}^{-1}$ (Figure S3c). This indicates the uniformity of the hot spots created and homogeneous distribution of the target molecule at the hot spots, which is crucial for generating a reproducible SERS signal. ${ }^{1,3}$
The reproducibility of the as-prepared SERS substrates was also evaluated by acquiring SERS spectra of CV $\left(1 \times 10^{-7} \mathrm{M}\right)$ (Figure S4a) dropped on triplicate sets of AuNP superstructures and triplicate acquisition for each replicate set. The result showed similar and consistent $(\mathrm{RSD}=12.4 \%)$ SERS signal intensification at $1621 \mathrm{~cm}^{-1}$ (Figure S4b), revealing good reproducibility of the SERS substrates. The long-time stability was evaluated by acquiring SERS spectra of CV-loaded substrates after five months of storage at room temperature (Figure S5), in which minimal variation in signal intensity was observed, revealing long-term stability of the substrates. The microstructure of the AuNP superstructures showed high stability without a noticeable change in morphology after five months of storage at room temperature, which is ascribed to the strong interaction between irreversibly aggregated AuNPs. Thus, the substrates can be used after five months, which is convenient for real-life application.

3.6. Application of the SERS Substrates for Detection of Thiram. The high sensitivity of the as-prepared substrates encourages us to further apply it for detection of trace Thiram pesticide from apple fruit surface. Thiram, a dithiocarbamate fungicide widely used in agriculture to prevent foodstuffs from fungal diseases, poses a risk to human health due to excessive use. ${ }^{38}$ This demands the development of a facile method for monitoring trace Thiram in environmental samples.

The feasibility of utilizing AuNP superstructures for SERS detection of Thiram was tested both in standard solution and eluted from apple surface. Figure 6a shows the SERS spectra of series concentrations $\left(1 \times 10^{-4} \mathrm{M}\right.$ to $\left.1 \times 10^{-7} \mathrm{M}\right)$ of Thiram in ethanol solution, in which characteristic Raman bands at 556, 1145,1380 , and $1510 \mathrm{~cm}^{-1}$ were observed. The observed peaks agreed with that reported in the literature, ${ }^{39}$ where the peak at $556 \mathrm{~cm}^{-1}$ is attributed to $v(\mathrm{SS})$ and that at $1145 \mathrm{~cm}^{-1}$ is associated with $\rho\left(\mathrm{CH}_{3}\right)$ coupled with $v(\mathrm{CN})$, the one at 1380 $\mathrm{cm}^{-1}$ is assigned to $\delta_{\mathrm{s}}\left(\mathrm{CH}_{3}\right)$ coupled with $v(\mathrm{CN})$, and that at $1510 \mathrm{~cm}^{-1}$ corresponds to $v(\mathrm{CN}), \delta\left(\mathrm{CH}_{3}\right)$, and $\rho\left(\mathrm{CH}_{3}\right)$. As can be seen from Figure 6, the Raman signal at $556 \mathrm{~cm}^{-1}$ assigned to $v(\mathrm{SS})$ mode is weak, while the one at $1380 \mathrm{~cm}^{-1}$ $\left(\delta \mathrm{s}\left(\mathrm{CH}_{3}\right)\right.$ coupled $\left.v(\mathrm{C}-\mathrm{N})\right)$ was strongly increased. This was in good agreement with previous reports and was ascribed to the binding of Thiram to the AuNP surface through the $S$ atom. ${ }^{40}$ As reported elsewhere, Thiram strongly interacts with metal nanoparticles ( $\mathrm{Ag}$ and $\mathrm{Au}$ ) and forms a bidentate thiolate bond with two dimethyl residues formed by cleavage of the S- 
$S$ bond, hence strongly adsorbing onto the surface of AuNPs, benefiting SERS detection of trace Thiram. ${ }^{40}$ Figure 6 also shows that the intensity of main Raman bands at 556, 1145, 1380 , and $1510 \mathrm{~cm}^{-1}$ increased with Thiram concentration and was distinguishable at a concentration as low as $1 \times 10^{-7} \mathrm{M}$. A plot of the SERS signal intensity at $1380 \mathrm{~cm}^{-1}$ against the logarithm of Thiram concentration revealed a linear relationship (inset of Figure 6a). The reproducibility of the SERS substrates for the detection of Thiram was evaluated using triplicate batches of the substrates prepared under the same conditions. The collected SERS signals showed variation in signal intensity with RSD value of $10.4 \%$ (Figure S6), indicating good reproducibility of the method.

The real-life application of the as-prepared substrates for SERS detection was verified by detecting Thiram eluted from apple fruit peels. The collected SERS spectra of different concentrations of eluted Thiram were shown in Figure $6 \mathrm{~b}$. However, there was no Thiram Raman signal observed for blank samples. The LOD for Thiram, for spiked samples, was found to be $0.28 \mathrm{ng} / \mathrm{cm}^{2}$. The obtained recovery values of the Thiram ranged from $63 \%$ to $93 \%$. Although the extraction efficiency is not very high for accurate extraction and detection of the exact Thiram concentration in apple peel, the method still can be fairly used for practical SERS detection because a limit of detection (LOD) as low as $0.28 \mathrm{ng} / \mathrm{cm}^{2}$ was estimated, which is much lower than the permissible level for apple peels $\left(2 \mu \mathrm{g} / \mathrm{cm}^{2}\right){ }^{40}$ Therefore, the SERS detection of Thiram at such low concentrations $\left(0.28 \mathrm{ng} / \mathrm{cm}^{2}\right)$ reveals the potential of the SERS platform for monitoring food safety and human health.

\section{CONCLUSIONS}

A new method for assembling of AuNP superstructures as efficient SERS substrates was developed by simply freezingthawing AuNP colloid. The suitability of AuNP superstructures as SERS substrates was demonstrated by its excellent SERS performance with uniformly distributed hot spots throughout the large surface area of the superstructures. The SERS activity increased with the particle size of AuNPs (18.3 \pm 1.9 to $40.5 \pm$ $3.6 \mathrm{~nm}$ ) in the assemblies as well as the aggregate size of the prepared AuNP superstructures. The high sensitivity and reproducibility of the produced cost-efficient SERS active substrates were ascribed to the formation of uniformly distributed high-density hot spots on the superstructures. The substrates showed long-term stability with minimal variation in signal intensity over five months of storage without a noticeable change in the morphology of the prepared AuNP superstructures. Moreover, the as-prepared SERS substrates showed practicability by detecting trace Thiram eluted from apple fruit at a concentration much lower than the permissible limits for apple peels. The current method is decisively advantageous because of its utilities such as low cost, simplicity, and being a promising strategy of technique miniaturization.

\section{ASSOCIATED CONTENT}

\section{S Supporting Information}

The Supporting Information is available free of charge on the ACS Publications website at DOI: 10.1021/acsami.6b15378.

TEM, SERS, and Raman mapping images and estimation of SERS enhancement factor (PDF)

\section{AUTHOR INFORMATION}

\section{Corresponding Author}

*E-mail: jfliu@rcees.ac.cn. Fax: +86-10-62849192.

ORCID 우

Rui Liu: 0000-0001-6353-6193

Jing-Fu Liu: 0000-0001-7134-7026

\section{Notes}

The authors declare no competing financial interest.

\section{ACKNOWLEDGMENTS}

This work was supported by the National Key Research and Development Program of China (2016YFA0203102), the Strategic Priority Research Program of the Chinese Academy of Sciences (XDB14020101), and the National Natural Science Foundation of China (21227012). D.B. and M.A. acknowledge the support of CAS-TWAS President's Fellowship for their Ph.D. study.

\section{REFERENCES}

(1) Liu, R.; Sun, J. F.; Cao, D.; Zhang, L. Q.; Liu, J. F.; Jiang, G. B. Fabrication of Highly-Specific SERS Substrates by Co-Precipitation of Functional Nanomaterials During the Self-Sedimentation of Silver Nanowires into a Nanoporous Film. Chem. Commun. 2015, 51, 13091312.

(2) Huang, J.; Ma, D.; Chen, F.; Bai, M.; Xu, K.; Zhao, Y. Ag Nanoparticles Decorated Cactus-Like Ag Dendrites/Si Nanoneedles as Highly Efficient 3d Surface-Enhanced Raman Scattering Substrates toward Sensitive Sensing. Anal. Chem. 2015, 87, 10527-10534.

(3) Chen, H.-Y.; Lin, M. H.; Wang, C. Y.; Chang, Y. M.; Gwo, S. Large-Scale Hot Spot Engineering for Quantitative SERS at the SingleMolecule Scale. J. Am. Chem. Soc. 2015, 137, 13698-13705.

(4) Bi, L.; Dong, J.; Xie, W.; Lu, W.; Tong, W.; Tao, L.; Qian, W. Bimetallic Gold-Silver Nanoplate Array as a Highly Active SERS Substrate for Detection of Streptavidin/Biotin Assemblies. Anal. Chim. Acta 2013, 805, 95-100.

(5) Liu, R.; Liu, J. F.; Zhang, Z. M.; Zhang, L. Q.; Sun, J. F.; Sun, M. T.; Jiang, G. B. Submonolayer-Pt-Coated Ultrathin Au Nanowires and Their Self-Organized Nanoporous Film: SERS and Catalysis Active Substrates for Operando SERS Monitoring of Catalytic Reactions. J. Phys. Chem. Lett. 2014, 5, 969-975.

(6) Zhang, Z. M.; Liu, J. F.; Liu, R.; Sun, J. F.; Wei, G. H. Thin Layer Chromatography Coupled with Surface-Enhanced Raman Scattering as a Facile Method for on-Site Quantitative Monitoring of Chemical Reactions. Anal. Chem. 2014, 86, 7286-7292.

(7) Tang, J. J.; Sun, J. F.; Lui, R.; Zhang, Z. M.; Liu, J. F.; Xie, J. W. New Surface-Enhanced Raman Sensing Chip Designed for on-Site Detection of Active Ricin in Complex Matrices Based on Specific Depurination. ACS Appl. Mater. Interfaces 2016, 8, 2449-2455.

(8) Liu, R.; Liu, J. F.; Zhou, X. X.; Sun, M. T.; Jiang, G. B. Fabrication of a $\mathrm{Au}$ Nanoporous Film by Self-Organization of Networked Ultrathin Nanowires and Its Application as a Surface-Enhanced Raman Scattering Substrate for Single-Molecule Detection. Anal. Chem. 2011, 83, 9131-9137.

(9) Jiang, J.; Ou-Yang, L.; Zhu, L.; Zou, J.; Tang, H. Novel One-Pot Fabrication of Lab-on-a-Bubble@Ag Substrate without CouplingAgent for Surface Enhanced Raman Scattering. Sci. Rep. 2014, 4, 3942.

(10) Fateixa, S.; Nogueira, H. I. S.; Trindade, T. Hybrid Nanostructures for SERS: Materials Development and Chemical Detection. Phys. Chem. Chem. Phys. 2015, 17, 21046-21071.

(11) Novak, J. P.; Brousseau, L. C.; Vance, F. W.; Johnson, R. C.; Lemon, B. I.; Hupp, J. T.; Feldheim, D. L. Nonlinear Optical Properties of Molecularly Bridged Gold Nanoparticle Arrays. J. Am. Chem. Soc. 2000, 122, 12029-12030.

(12) Radziuk, D.; Moehwald, H. Prospects for Plasmonic Hot Spots in Single Molecule SERS Towards the Chemical Imaging of Live Cells. Phys. Chem. Chem. Phys. 2015, 17, 21072-21093. 
(13) Ghosh, S. K.; Pal, T. Interparticle Coupling Effect on the Surface Plasmon Resonance of Gold Nanoparticles: From Theory to Applications. Chem. Rev. 2007, 107, 4797-4862.

(14) Majumdar, D.; Singha, A.; Mondal, P. K.; Kundu, S. DNAMediated Wirelike Clusters of Silver Nanoparticles: An Ultrasensitive SERS Substrate. ACS Appl. Mater. Interfaces 2013, 5, 7798-7807.

(15) Sun, L.; Zhao, D.; Ding, M.; Xu, Z.; Zhang, Z.; Li, B.; Shen, D. Controllable Synthesis of Silver Nanoparticle Aggregates for SurfaceEnhanced Raman Scattering Studies. J. Phys. Chem. C 2011, 115, 16295-16304.

(16) Yang, Y.; Shi, J.; Tanaka, T.; Nogami, M. Self-Assembled Silver Nanochains for Surface-Enhanced Raman Scattering. Langmuir 2007, 23, 12042-12047.

(17) Fang, Y.; Seong, N.-H.; Dlott, D. D. Measurement of the Distribution of Site Enhancements in Surface-Enhanced Raman Scattering. Science 2008, 321, 388-392.

(18) Taylor, R. W.; Lee, T. C.; Scherman, O. A.; Esteban, R.; Aizpurua, J.; Huang, F. M.; Baumberg, J. J.; Mahajan, S. Precise Subnanometer Plasmonic Junctions for SERS within Gold Nanoparticle Assemblies Using Cucurbit[N]Uril "Glue. ACS Nano 2011, 5, 3878-3887.

(19) Dey, P.; Blakey, I.; Thurecht, K. J.; Fredericks, P. M. Hyperbranched Polymer-Gold Nanoparticle Assemblies: Role of Polymer Architecture in Hybrid Assembly Formation and SERS Activity. Langmuir 2014, 30, 2249-2258.

(20) Kim, P. Y.; Oh, J.-W.; Nam, J.-M. Controlled Co-Assembly of Nanoparticles and Polymer into Ultralong and Continuous OneDimensional Nanochains. J. Am. Chem. Soc. 2015, 137, 8030-8033.

(21) Zhang, H.; Fung, K. H.; Hartmann, J.; Chan, C. T.; Wang, D. Controlled Chainlike Agglomeration of Charged Gold Nanoparticles Via a Deliberate Interaction Balance. J. Phys. Chem. C 2008, 112, 16830-16839.

(22) Huang, T.; Meng, F.; Qi, L. Facile Synthesis and OneDimensional Assembly of Cyclodextrin-Capped Gold Nanoparticles and Their Applications in Catalysis and Surface-Enhanced Raman Scattering. J. Phys. Chem. C 2009, 113, 13636-13642.

(23) Schwartzberg, A. M.; Grant, C. D.; Wolcott, A.; Talley, C. E.; Huser, T. R.; Bogomolni, R.; Zhang, J. Z. Unique Gold Nanoparticle Aggregates as a Highly Active Surface-Enhanced Raman Scattering Substrate. J. Phys. Chem. B 2004, 108, 19191-19197.

(24) Liu, J.; White, I.; DeVoe, D. L. Nanoparticle-Functionalized Porous Polymer Monolith Detection Elements for Surface-Enhanced Raman Scattering. Anal. Chem. 2011, 83, 2119-2124.

(25) Shen, X.; Chen, L.; Li, D.; Zhu, L.; Wang, H.; Liu, C.; Wang, Y.; Xiong, Q.; Chen, H. Assembly of Colloidal Nanoparticles Directed by the Microstructures of Polycrystalline Ice. ACS Nano 2011, 5, 84268433.

(26) Nye, J. F. Thermal Behaviour of Glacier and Laboratory Ice. J. Glaciol. 1991, 37, 401-413.

(27) Frens, G. Controlled Nucleation for Regulation of Particlesize in Monodisperse Gold Suspensions. Nature, Phys. Sci. 1973, 241, 20-22.

(28) Lewis, D. J.; Day, T. M.; MacPherson, J. V.; Pikramenou, Z. Luminescent Nanobeads: Attachment of Surface Reactive Eu(III) Complexes to Gold Nanoparticles. Chem. Commun. 2006, 1433-1435.

(29) Mader, H. Observations of the Water-Vein System in Polycrystalline Ice. J. Glaciol. 1992, 38, 333-348.

(30) Sugawa, K.; Akiyama, T.; Tanoue, Y.; Harumoto, T.; Yanagida, S.; Yasumori, A.; Tomita, S.; Otsuki, J. Particle Size Dependence of the Surface-Enhanced Raman Scattering Properties of Densely Arranged Two-Dimensional Assemblies of $\mathrm{Au}$ (Core)- $\mathrm{Ag}$ (Shell) Nanospheres. Phys. Chem. Chem. Phys. 2015, 17, 21182-21189.

(31) Stamplecoskie, K. G.; Scaiano, J. C.; Tiwari, V. S.; Anis, H. Optimal Size of Silver Nanoparticles for Surface-Enhanced Raman Spectroscopy. J. Phys. Chem. C 2011, 115, 1403-1409.

(32) Zhang, K.; Zhao, J.; Xu, H.; Li, Y.; Ji, J.; Liu, B. Multifunctional Paper Strip Based on Self-Assembled Interfacial Plasmonic Nanoparticle Arrays for Sensitive SERS Detection. ACS Appl. Mater. Interfaces 2015, 7, 16767-16774.
(33) Joseph, V.; Matschulat, A.; Polte, J.; Rolf, S.; Emmerling, F.; Kneipp, J. SERS Enhancement of Gold Nanospheres of Defined Size. J. Raman Spectrosc. 2011, 42, 1736-1742.

(34) Seney, C. S.; Gutzman, B. M.; Goddard, R. H. Correlation of Size and Surface-Enhanced Raman Scattering Activity of Optical and Spectroscopic Properties for Silver Nanoparticles. J. Phys. Chem. C 2009, 113, 74-80.

(35) Hu, Z.; Liu, Z.; Li, L.; Quan, B.; Li, Y.; Li, J.; Gu, C. Wafer-Scale Double-Layer Stacked $\mathrm{Au} / \mathrm{Al}_{2} \mathrm{O}_{3} @ \mathrm{Au}$ Nanosphere Structure with Tunable Nanospacing for Surface-Enhanced Raman Scattering. Small 2014, 10, 3933-3942.

(36) Sztainbuch, I. W. The Effects of Au Aggregate Morphology on Surface-Enhanced Raman Scattering Enhancement. J. Chem. Phys. 2006, 125, 124707.

(37) Yang, M.; Chen, G.; Zhao, Y.; Silber, G.; Wang, Y.; Xing, S.; Han, Y.; Chen, H. Mechanistic Investigation into the Spontaneous Linear Assembly of Gold Nanospheres. Phys. Chem. Chem. Phys. 2010, 12, 11850-11860.

(38) Khlebtsov, B. N.; Khanadeev, V. A.; Panfilova, E. V.; Bratashov, D. N.; Khlebtsov, N. G. Gold Nanoisland Films as Reproducible SERS Substrates for Highly Sensitive Detection of Fungicides. ACS Appl. Mater. Interfaces 2015, 7, 6518-6529.

(39) Saute, B.; Narayanan, R. Solution-Based Direct Readout Surface Enhanced Raman Spectroscopic (SERS) Detection of Ultra-Low Levels of Thiram with Dogbone Shaped Gold Nanoparticles. Analyst 2011, 136, 527-532.

(40) Liu, B.; Han, G.; Zhang, Z.; Liu, R.; Jiang, C.; Wang, S.; Han, M.-Y. Shell Thickness-Dependent Raman Enhancement for Rapid Identification and Detection of Pesticide Residues at Fruit Peels. Anal. Chem. 2012, 84, 255-261. 